Horizons philosophiques

\title{
Raison et morale chez Montaigne : Marcel Conche face à André Comte-Sponville
}

\section{Sébastien Charles}

Volume 9, numéro 1, automne 1998

Médiations

URI : https://id.erudit.org/iderudit/801089ar

DOI : https://doi.org/10.7202/801089ar

Aller au sommaire du numéro

Éditeur(s)

Collège Édouard-Montpetit

ISSN

1181-9227 (imprimé)

1920-2954 (numérique)

Découvrir la revue

\section{Citer cet article}

Charles, S. (1998). Raison et morale chez Montaigne : Marcel Conche face à André Comte-Sponville. Horizons philosophiques, 9(1), 17-31.

https://doi.org/10.7202/801089ar d'utilisation que vous pouvez consulter en ligne.

https://apropos.erudit.org/fr/usagers/politique-dutilisation/ 


\section{RAISON ET MORALE CHEZ MONTAIGNE : MARCEL CONCHE FACE À ANDRÉ COMTE-SPONVILLE1}

Que Montaigne reste l'apanage des littéraires, c'est ce que de nombreux philosophes ont voulu, en toute conscience, ne voyant en lui, sur le plan philosophique, qu'une source possible de la subjectivité cartésienne. Mais ce temps semble révolu depuis que deux philosophes français², et non des moindres, se sont penchés sur les Essais où ils ont découvert pour l'un un philosophe authentique ${ }^{3}$ et pour l'autre un «sage à sa façon, et plus philosophe que nos sophistes, et plus sage que les sages4". Et d'ailleurs le Montaigne et la philosophies de Marcel Conche et le "Je ne suis pas philosophe» : Montaigne et la philosophie6 d'André Comte-Sponville sont des titres qui parlent d'eux-mêmes. Ainsi donc Montaigne appartient pour eux de fait

1. Ce texte, qui a fait l'objet d'une communication devant la Société Canadienne d'Études de la Renaissance lors du Congrès des Sociétés Savantes à SaintJohn's (Terre-Neuve) en juin 1997, a obtenu une mention honorifique décernée à la seconde meilleure communication étudiante tant pour sa clarté que pour son éloquence.

2. Pour une brève présentation de Marcel Conche, cf. notre "Pour une nouvelle philosophie française : Marcel Conche", Bulletin de la Société de Philosophie du Québec, 24,1 (hiver 1998), p.7-11; pour Comte-Sponville, cf. notre «Pour une nouvelle philosophie française : André Comte-Sponville", Bulletin de la Société de Philosophie du Québec, 23,4 (automne 1997), p10-11.

3. Marcel Conche, Préface à Pierre Leschemelle, Montaigne, le badin de la farce, Paris, Imago, 1995, p. 9 : “Des philosophes modernes, Montaigne n'est sans doute pas le plus grand, mais il est le plus authentique : lui qui ne se dit pas philosophe, a le mieux su que la philosophie est impossible comme science, et qu'en dernière analyse le scepticisme est le vrai." Cf. aussi l'article "Montaigne" dans le Dictionnaire des philosophes de Denis Huisman, Paris : P.U.F., 1984, p. 1844 : "Si le philosophe est celui qui, ayant mis en doute ce qui nous est donné par le sens commun ou par les systèmes philosophiques comme "réel" ou "vrai", tient un discours cohérent au sujet de tout ce qui lui semble, à lui, "vrai» ou "réel», Montaigne est bien, de plein droit, philosophe."

4. André Comte-Sponville, Préface au chapitre IX du livre III des Essais : De la vanité, Paris : Rivages, 1989, p. 12. Cf. aussi "Montaigne ou la philosophie vivante", Une éducation philosophique, Paris : P.U.F., coll. "Perspectives critiques", 1989, p. 236 : "Si Montaigne est peu lu, c'est qu'il est philosophe, ce qui effraie les ignorants, et point auteur de système, ce qui déroute les savants." 
au penser philosophique et il en est même un sommet incontournable par sa lucidité à l'égard du monde et de l'homme.

Philosophe, soit, il faut en convenir. Mais encore faut-il s'entendre sur les termes. Selon Conche, «l'œuvre principale du philosophe, celle qui atteste qu'il n'a pas philosophé pour la galerie mais pour de bon, c'est-à-dire pour lui, pour devenir "meilleur et plus sage», cette œuvre est d'abord le philosophe lui-même7». Singularité du philosophe, singularité de Montaigne. Ce qui fait de Montaigne un philosophe avant tout c'est qu'il a cherché, selon la belle formule d'André ComteSponville, à "penser sa vie et vivre sa pensée8". C'est là l'activité authentique du philosophe d'où il tire sa joie ou, comme dirait Montaigne, "son éjouissance constante ${ }^{9}$ " (I, XXVI, 161; PI. 160). Cela suppose, et nos deux lecteurs de Montaigne le voient bien, que l'authentique philosophe ne saurait être dogmatique puisque tout système clôt sur ellemême la pensée et fait de la philosophie une idéologie'10. C'est ce que Montaigne ne pouvait accepter : «'admiration [l'étonnement] est fondement de toute philosophie, l'inquisition [la recherche] le progrès, l'ignorance le bout" (III, XI, 1030; PI. 1008). D'où son scepticisme qui n'est autre que le refus de toute certitude théorique en philosophie. Qui ne voit que sur ce point Montaigne s'éloigne de ses maîtres antiques qu'il admire et dont l'admiration le porte à les excuser pour leur

5. Marcel Conche, Montaigne et la philosophie, Treffort, Éditions de Mégare, 1987; $2^{\mathbf{e}}$ éd., 1992; réimpression augmentée aux P.U.F., coll. "Perspectives critiques", 1996. C'est cette dernière édition que nous citerons.

6. "Je ne suis pas philosophe": Montaigne et la philosophie, Paris : Honoré Champion, coll. "Etudes montaignistes", 1993.

7. Marcel Conche, Montaigne et la philosophie, p. 107.

8. André Comte-Sponville, L'amour la solitude, Vénissieux, Paroles d'Aube, 1992, p. 13. Même formule dans Une éducation philosophique, Paris : P.U.F., coll. "Perspectives critiques", 1989, p. 344. André Comte-Sponville défend cette expression dans "Philosopher", L'express, semaine du 15 au 21 février 1996.

9. Nos références aux Essais renvoient à l'édition Villey, revue et corrigée par V.-

L. Saulnier, Paris, P.U.F., 1978 dont nous avons modernisé l'orthographe. Pour plus de commodité, nous citons parallèlement la pagination de l'édition faite par Maurice Rat et parue en 1962 dans la "Bibliothèque de la Pléiade".

10. D'où le mot célèbre de Shaftesbury : "Le moyen le plus sensé pour devenir fou est de passer par un système". 
rigidité systématique qui ne peut être, à ses yeux, que jeu : “Je ne me persuade pas aisément qu'Épicure, Platon et Pythagore nous aient donné pour argent comptant leurs Atomes, leurs Idées et leurs Nombres. Ils étaient trop sages pour établir leurs articles de foi de chose si incertaine et si débattable" (II, XII, 511; PI. 491-492). Sagesse de Montaigne qui attribue sa propre modération à ceux qu'il lit. Scepticisme de Montaigne aussi qui remet en question les postulats philosophiques de ses devanciers. Pourtant cela ne l'amène pas à basculer dans le nihilisme ou la sophistique. Montaigne ne refuse pas de croire qu'il y ait une vérité en soi, mais seulement qu'elle nous soit accessible hic et nunc.

C'est ce que Marcel Conche et André Comte-Sponville mettent parfaitement en lumière et la dette philosophique du second à l'égard du premier est sur ce point indiscutable. Marcel Conche, le "Maître11», nous indique que toute essence n'est pas absente de la philosophie montanienne puisque Dieu est, et ce pour l'éternité. Seulement, il ne nous est pas donné de le connaître et notre condition d'êtres sublunaires nous interdit de prétendre à quelque vérité théorique objective. L'élève nous apprend que par cette position même Montaigne échappe à la fois aux contradictions logiques inhérentes au nihilisme (si rien n'est vrai, il n'est pas vrai que rien ne soit vrai) et à la sophistique (si on ne sait rien, comment peut-on le savoir?) : «Pour sceptique qu'il soit, Montaigne n'a jamais cessé, non pas tant de chercher la vérité, que de s'y soumettre et de l'aimer, là où il la trouvait, et même, c'est tout l'esprit du scepticisme, là où il ne la trouvait pas. C'est ce qui distingue sa philosophie de la sophistique, et qui le rattache à Socrate, en effet, davantage qu'à ceux que Socrate combattait. II s'agit d'aimer le vrai, fût-ce en son absence, et de s'y soumettre dès lors qu'il apparaît ou semble apparaître ${ }^{12 » . ~ L e ~ " Q u e ~ s a i s-j e ? » ~ d e ~ M o n t a i g n e ~ n ' e s t ~}$

11. C'est le terme même employé avec déférence par André Comte-Sponville à la page 7 de sa préface de l'Orientation philosophique de Marcel Conche parue aux P.U.F., dans la collection "Perspectives critiques" en 1990.

12. André Comte-Sponville, "Je ne suis pas philosophe" : Montaigne et la philosophie, p. 42-43. 
donc pas à prendre dans un sens absolu mais relatif. Si «nous n'avons aucune communication à l'être» (II, XII, 601; PI. 586), comme le rappelle Montaigne, et à sa suite ses deux exégètes, cela n'implique pas pour autant un héraclitéisme total. Cela signifie simplement que l'essence des choses nous échappe, tout comme celle de Dieu, du Vrai, du Beau et du Bien. Mais cela n'altère en rien leur existence; par contre, le statut cognitif humain est par là même dépossédé de sa prétention à la certitude : "Dès lors, si la raison n'a d'appui que dans l'apparence [...] ce à quoi il nous faut renoncer, c'est au savoir luimême, et à fonder la sagesse sur le savoir'13". C'est là accepter l'oracle de Delphes qui nous renvoie à notre singularité et qui nous apprend en outre la relativité de toute connaissance théorique. L'essence de l'homme nous est tout aussi inaccessible que l'essence de Dieu. Notre conscience naturellement singulière est soumise à son temps et aux opinions dont il est fait, comme elle l'est à la nature qui nous fait et nous défait. "Nature s'est obligée à ne rien faire autre, qui ne fût dissemblable» (III, XIII, 1065; PI. 1042). D'où la diversité des corps, sans doute, mais aussi des esprits, et donc des jugements et des opinions. Une philosophie fondée sur un tel relativisme, avantageuse en théorie parce que stimulante, ne pourrait, en pratique, qu'être dangereuse en permettant la justification de bien des horreurs. C'est ce que Montaigne, en vertu de son humanisme pratique, n'aurait pu accepter. Marcel Conche et André Comte-Sponville s'y refusent également, et c'est pourtant là que leur lecture de Montaigne achoppe.

Au départ, pourtant, leur lecture est parallèle. Ils voient bien tous deux que le scepticisme de Montaigne peut donner lieu à des dérives nihilistes. Si Montaigne reconnaît en effet que les coutumes humaines sont relatives à l'espace et au temps, comment prétendre qu'une morale singulière puisse les juger universellement? Et si Montaigne va plus loin en montrant que les lois prétendument naturelles sont elles aussi relatives, où trouver un fondement objectif pour une morale valant en droit

13. Marcel Conche, Montaigne ou la conscience heureuse, Paris, Seghers, 1964; $3^{e}$ éd., 1970; $4^{e}$ éd., Éd. de Mégare, 1992, p. 56. 
comme en fait pour tous? Or, n'est-ce pas ce que dit Montaigne? Relativité des lois naturelles : «il n'en y a une seule qui ne soit contredite et désavouée, non par une nation, mais par plusieurs" (II, XII, 580 ; PI. 564). Relativité des lois civiles : "Quelle bonté est-ce que je voyais hier en crédit, et demain plus, et que le trait d'une rivière fait crime? Quelle vérité que ces montagnes bornent, qui est mensonge au monde qui se tient au-delà?» (II, XII, 579; PI. 563). L'homme est le fruit d'une nature et d'une culture qui le tiennent fermement. Si, selon l'expression de Jean Borella, "la nature de l'homme, c'est sa culture», on voit mal comment il pourrait lui échapper. Et pourtant une porte de sortie reste envisageable, qui est celle de notre conscience, et qui fait toute notre valeur. La conscience s'offre comme remède possible au nihilisme pratique. Mais peut-on en rester là quand Montaigne semble indiquer que toute conscience est à jamais déterminée par la spatiotemporalité des existants humains : "Les lois de la conscience, que nous disons naître de nature, naissent de la coutume : chacun ayant en vénération interne les opinions et mœurs approuvées et reçues autour de lui, ne s'en peut déprendre sans remords, ni s'y appliquer sans applaudissement" (I, XXIII, 115; PI. 114). Rechercher dès lors l'universel dans la bigarrure des opinions semble relever de l'exploit. C'est ce que vont pourtant tenter de faire, à leur manière, Marcel Conche et André Comte-Sponville qui refusent de lire un quelconque relativisme dans les Essais tout comme de voir dans Montaigne un nihiliste.

Pour Marcel Conche, il existe une conscience morale perceptible dans les Essais et qui nous permet de fonder la possibilité d'une morale universelle. Marcel Conche évoque une "voix intérieure" qui n'est pas sans rappeler le daimon de Socrate et qui nous permet de juger en conscience. Nul doute que ceux à qui cet honneur est rendu sont rares mais malgré l'influence tyrannique de la coutume sur tout homme on ne peut récuser leur existence. Ces hommes exceptionnels sont ceux qui ont su se tourner vers leur intériorité pour, à partir de là, 
juger en toute liberté14. Ce sont les sages : «Certes, il est possible de tenir son âme "en liberté et puissance de juger librement des choses" (I, XXIII, 118 ; PI. 117) et de les rapporter, malgré la coutume, "à la vérité et à la raison", mais cette possibilité appartient au sage. L'insensé, dont le jugement est malade, la liberté non libre, prisonnière de son propre mauvais vouloir, est, par là même, condamné à demeurer enfermé dans sa folie ${ }^{15}$ ". Pour légitimer son entreprise fondatrice, Conche s'appuie sur un passage célèbre du chapitre XXIII du livre I : "Qui voudra se défaire de ce violent préjudice [préjugé] de la coutume, il trouvera plusieurs choses reçues d'une résolution indubitable, qui n'ont appui qu'en la barbe chenue et rides de l'usage qui les accompagne ; mais, ce masque arraché, rapportant les choses à la vérité et à la raison, il sentira son jugement comme tout bouleversé, et remis pourtant en bien plus sûr état" (117; PI. 116). Cela renvoie chaque homme à sa propre conscience, si du moins il en est capable. C'est ce que croit Marcel Conche : "La raison n'est qu'individuelle, aujourd'hui comme au temps de Montaigne, et comme elle l'a toujours été. Elle n'est jamais collective. On n'atteint l'universel qu'en libérant d'abord la raison de l'emprise collective ${ }^{16 "}$. Montaigne est la preuve vivante qu'une telle libération est possible : «J'ai mes lois, écritil, et ma cour pour juger de moi, et m'y adresse plus qu'ailleurs" (III, II, 807; PI. 785). La conscience est ce qui nous fait être ce que nous sommes face à un monde soumis au branle permanent. On comprend alors le respect sacré que Montaigne accorde à la parole donnée, seul gage de notre permanence dans l'être : "Le fondement de mon être et de mon identité est purement moral : il se trouve dans la fidélité à la foi que je me suis jurée à moi-même. Je ne suis pas réellement le même qu'hier; je ne suis le même que parce que je m'avoue le même,

14. Marcel Conche pense-t-il ici à La Boétie? La chose est probable même si le célèbre ami de Montaigne n'est pas nommé. En tout cas, sa manière de traiter du thème de la liberté dans son Discours de la servitude volontaire lui mériterait une place dans ce passage.

15. Ibid., p. 38.

16. Marcel Conche, "Montaigne me manque", Montaigne philosophe, textes réunis par André Comte-Sponville, Revue internationale de philosophie, $n^{0} 181,2$, 1992, p. 167. Repris dans Montaigne et la philosophie. 
parce que je prends à mon compte un certain passé comme le mien, et parce que j'entends, dans l'avenir, reconnaître mon engagement présent comme toujours le mien ${ }^{17}$ ». On est bien loin là du Gygès de Platon qui, se sachant invisible, perpétrait les crimes qu'il lui plaisait de commettre. Mais ce que Montaigne nous montre, c'est que nous n'échappons pas à notre propre conscience pour qui nous sommes toujours visibles ${ }^{18}$. Qui, mieux que nous, nous connaît? Notre conscience n'est-elle pas celle qui sonde, à sa façon, les reins et les cœurs? Elle est donc ce qui nous oblige à accomplir dignement notre devoir. Ainsi Montaigne, tout comme Abélard19 d'ailleurs, ne peut que privilégier une morale de l'intériorité, et donc de l'intention. Morale de l'intention, morale de la volonté, qui trouve sa récompense dans le jugement que chacun porte sur soi.

Marcel Conche n'en reste pas à ce simple constat, auquel André Comte-Sponville se rattache pleinement. II prétend en outre que la morale montanienne est aussi une morale fondée sur la raison, et par là même universelle. Bien que Montaigne dénigre la raison dans sa prétention à l'objectivité, Marcel Conche nous montre qu'il la valorise dans son usage critique en théorie comme dans son usage normatif en pratique. D'après Conche, si Montaigne se déclare uniquement esclave de la raison (III, I, 794; PI. 772), c'est parce qu'elle «fonde notre devoir20". C'est aussi parce qu'elle est avant tout raison discursive et qu'elle permet aux hommes d'échanger entre eux leur parole, de se respecter et de s'associer pour fonder une communauté politique viable. Et, en sa limite extrême, elle nous permet en tant que raison morale de poser "des exigences et des lois morales universelles qui s'imposent à nous ${ }^{21}$ ». Cet

17. Montaigne et la philosophie, p. 119.

18. Cf. ce que dit à ce propos André Comte-Sponville d'Arsène Lupin, sur ce point bien proche de Montaigne, dans "La morale d'Arsène Lupin ou Arsène Lupin contre Kant", Arsène Lupin gentilhomme-philosopheur, Paris : Éditions du félin, 1996, spécialement p. 53-56.

19. Cf. Sébastien Charles, Le rapport à la vérité chez Pierre Abélard et saint Bernard, Nancy, inédit, 1995, p. 78-85 et Gilbert Boss, "Le combat d'Abélard", Cahiers de civilisation médiévale, $\mathrm{n}^{\circ}$ 1, 1988, p. 17-27.

20. Montaigne et la philosophie, p. 125.

21. Ibid., p. 156. C'est aussi la position de l'Abbé Gierczynski, "La problématique morale dans les Essais de Montaigne", Bulletin de la Société des Amis de Montaigne, janvier-juin 1981, p. 29-42. 
universalisme, Marcel Conche le fonde sur la parole que les hommes de bonne foi échangent entre eux en opérant une réduction dialogique qui met de côté leurs particularismes pour pouvoir atteindre au vrai et donc à l'universel : "La morale se fonde non sur telle ou telle croyance, religion ou système, mais sur cet absolu qu'est la relation de l'homme à l'homme dans le dialogue22».

C'est sur ce point que l'élève se sépare du maître et refuse de lire une telle possibilité fondatrice chez Montaigne. André Comte-Sponville n'abolit pas pour autant le rôle capital de la conscience singulière, mais il lui refuse tout universalisme absolu. II reconnaît, avec Marcel Conche, que la conscience montanienne est toute d'intention et donc de volonté. Mais elle n'est que cela, à savoir la morale d'une volonté particulière, celle d'un individu qui la pense, la veut et la vit. Cela ruine toute idée d'absoluité morale et Marcel Conche s'en est offusqué, voyant là un relativisme culturel pouvant mener directement à une tolérance généralisée qui n'aurait rien à opposer aux intolérants ${ }^{23}$. Que le relativisme culturel absolu mène au nihilisme, cela est malheureusement vrai, et l'histoire nous l'a prouvé avec le nazisme. Mais est-ce vraiment la lecture qu'André Comte-Sponville fait de Montaigne? Nous ne le croyons pas. Bien sûr, André Comte-Sponville refuse de voir dans la conscience humaine un fondement possible pour une morale universelle, préférant plutôt parler de principe : "Principe, non fondement. Car si l'on entend par fondement de quoi légitimer une morale universelle ou absolue, c'est quelque chose [...] qu'on ne saurait guère trouver dans les Essais24». Et de montrer que ni la nature ni la raison ne parviennent à

22. Marcel Conche, Le fondement de la morale, P.U.F., coll. "Perspectives critiques", 1993, p. 2. Cela rappelle le "voile d'ignorance" rawlsien. Cf. Rawls, Théorie de la justice, Paris : Seuil, coll. "La couleur des idées", 1987, § 24, p. 168-174.

23. André Comte-Sponville refuse cette perspective à plusieurs reprises et notamment dans le Petit traité des grandes vertus, Paris : P.U.F., coll. "Perspectives critiques", 1995, où il analyse longuement cette vertu particulière qu'est la tolérance et montre qu'on ne peut bien sûr pas tout tolérer.

24. "Montaigne cynique? (Valeur et vérité dans les Essais)", Valeur et vérité, Études cyniques, Paris : P.U.F., coll. «Perspectives critiques», 1994, p. 70. 
légitimer quelque chose d'absolu. C'est là rejoindre une des critiques que Claude Lévi-Strauss adresse à Marcel Conche à qui il reproche de «croire que les droits de la conscience morale resteraient intacts chez Montaigne" et "qu'ils s'exerceraient dans et par l'usage de la raison ${ }^{25}$ ". Or, la raison, par sa nature versatile, ne saurait à elle seule donner un fondement philosophique à la morale. Reste que Montaigne, comme le souligne Marcel Conche, évoque parfois la possibilité de voir en la raison une garantie possible à un universalisme pratique. Mais il faut faire là attention à ne pas confondre deux points de vue différents sur la morale que Marcel Conche, aux yeux d'André Comte-Sponville, n'aurait pas distingués. Le premier porte sur la morale comme valeur universelle à penser, le second sur la morale comme devoir que chaque individu doit accomplir pour lui-même. Mais de l'un à l'autre, nul passage possible, et c'est cet abîme qui fait notre condition d'homme. Notre volonté est toujours singulière même si elle se propose un but universel : "Ce que j'entends par universalisable, c'est cela : un universel qui n'est pas celui de la vérité mais du désir, un universel qui est objet non de connaissance, mais de volonté, un universel qui n'est donné que dans l'action, et par elle, un universel qui ne vaut pour tous les hommes qu'autant que nous le défendons en effet, un universel qui n'est pas derrière nous, comme une caution ou une garantie, mais devant, comme un but ou un horizon. C'est le seul universel qui nous soit accessible, dans ces domaines, ou plutôt il ne l'est pas (nous n'en aurons jamais fini de le réaliser), mais il est offert à la fois à la pensée (comme valeur) et à l'action (comme but), ce dont nous ne saurions nous passer et qui doit nous suffire ${ }^{26}$ ".

C'est cela qu'André Comte-Sponville décèle chez. Montaigne en insistant à la fois sur son scepticisme théorique et sur son épicurisme pratique. Montaigne dépasse le solipsisme et le relativisme par sa volonté et son désir. Sa morale est bien évidemment singulière, puisqu'elle est celle de

25. Claude Lévi-Strauss, "En relisant Montaigne", Histoire de lynx, Paris : Plon, 1991, note de la p. 287.

26. "L'universel, singulièrement», Valeur et vérité, Études cyniques, p. 260. 
l'individu Montaigne, mais tout en valant universellement pour sa propre personne : "Sa morale ne vaut que pour lui, certes; mais, pour lui, elle vaut absolument. Elle relève de la foi, si l'on veut, mais au sens ancien, et non théologique du terme - au sens où la foi est fidélité à sa parole et à soi27». Mais on ne peut en rester à l'individu seul car ce serait manquer ce qui constitue son étoffe humaine : "La morale relève bien en cela de la fidélité, non seulement à sa propre parole, mais à ce que l'humanité a fait de soi et de nous, qu'elle nous lègue, et que nous lui devons ${ }^{28}$ ". Nous ne sommes donc toujours que face à une morale particulière, bien que déterminée par une histoire qui la constitue partiellement. Le jugement de tout homme est pris entre sa propre historicité et celle de sa communauté, voire de son espèce. Cela récuse définitivement tout rationalisme strict et toute idée de raison pure ${ }^{29}$. L'homme est à la fois raison et volonté et cette tension entre les deux, tension irréductible, lui est consubstantielle. C'est ce que Comte-Sponville nomme le cynisme, mouvement philosophique dont Montaigne est pour lui un représentant exemplaire. Est cynique celui qui sait que la vérité est sans valeur et que nos valeurs ne sont pas vraies. "C'est comme une plaie ouverte, mais qu'il est essentiel de ne pas refermer : car on ne pourrait alors que replier la valeur sur la vérité (comme font les dogmatiques) ou la vérité sur la valeur (comme font les sophistes), et c'en serait fini, dans les deux cas, de la liberté de l'esprit30». Mais cette plaie a son importance pour qui sait l'ausculter de près : elle permet la tolérance entre les diverses cultures qui peuplent notre monde. En effet,

27. "Montaigne cynique? (Valeur et vérité dans les Essais)", op. cit., p. 86.

28. Ibid., p. 88.

29. "La raison peut certes nous ouvrir à l'universel, mais non le fonder" écrivais-je dernièrement à Marcel Conche. Ce à quoi il me répondait que pour penser cela "il faudrait réfuter Kant - et [le] réfuter [...]. L'obligation de fonder la morale, disait-il en conclusion, ne s'impose qu'au philosophe. C'est une tâche philosophique». Tâche philosophique, sans doute, mais qui repose sur des a priori invérifiables, à savoir une universalité morale fondée sur une raison pure de type kantien. Mais peut-on réellement fonder une morale universalisante sur une raison non empirique? Kant a-t-il le droit - et même le pouvoir - de le faire? N'est-on pas là face à une illusion de la raison qui croit pouvoir se transcender elle-même? La raison pure n'est-elle pas la forme la plus subtile de ces antinomies qu'elle pense résoudre?

30. Ibid., p. 90. 
l'inexistence de norme absolue permet aux multiples communautés humaines de cohabiter : "Encore faut-il pour cela accepter les limites de l'humanité, et renoncer en conséquence à toute prétention à l'absolu : s'accepter homme, c'est aussi renoncer à être Dieu, et même à le connaître. Tout homme est alors l'égal de tout autre, ou du moins ne saurait légitimement juger à sa place. C'est là le seul universel qui nous reste, qui n'est d'ailleurs qu'une particularité très large, et qui a une portée surtout critique ou négative : il n'est autre que l'humanité elle-même, dans son existence de fait et dans l'acceptation de ses différences ${ }^{31}$ ».

Si le vrai nous est inaccessible hic et nunc et si nos valeurs sont relatives à notre condition d'êtres déterminés dans l'espace et le temps, on voit combien notre existence est, selon le mot de Marcel Conche, "tragique». C'est à chacun de juger universellement dans sa particularité sans normativité aucune. Comme me l'écrivait dernièrement André Comte-Sponville : "Au fond, ce qu'il faut chercher, c'est une morale qui soit à la fois relative et universalisable : une morale relativement universelle!". Montaigne n'est-il pas là la clef de l'énigme, lui qui nous apprend à accepter notre condition tragique et, si possible, «à faire bien l'homme»?

Au terme de cette mise en perspective de deux lectures possibles de Montaigne, il nous reste à comprendre les critiques que Marcel Conche adresse à son disciple et à évaluer leur portée polémique. Pour le maître, renoncer à affirmer l'universalité absolue d'une morale qui "est la même pour tous 32 ", c'est refuser le rôle fondateur de la raison humaine qui peut, à travers la conscience singulière d'un individu, dénoncer et condamner "des comportements, us et coutumes, que la collectivité tolère ou approuve [...]. En cela, la conscience morale individuelle pressent, prépare et annonce ce qui s'inscrira plus tard dans les institutions ${ }^{33}$ ». Montaigne est cette conscience singulière qui, quand elle s'oppose à la torture, au

31. Ibid., p. 95.

32. Montaigne et la philosophie, Avant-Propos, p. X.

33. Ibid. 
fanatisme ou à la barbarie soldatesque, vaut universellement. Sinon, qu'opposer au fanatisme si ce n'est un refus catégorique fondé rationnellement sur l'universel? Cette question, qui se veut décisive, pose problème car elle fait intervenir la raison comme normativité. Or, comme le montre judicieusement André Comte-Sponville, la raison n'a peut-être pas chez Montaigne «le rôle d'un fondement susceptible [...] d'ériger ses jugements de valeur en vérités universelles et nécessaires ${ }^{34}$ ". Marcel Conche en donne trop à la raison. Bien sûr, c'est elle qui nous ouvre à l'universel mais elle ne savait à elle seule être le tout de la morale. Elle est trop engluée dans une histoire singulière et collective pour s'en croire affranchie et par là fonder une morale universelle : "La raison est moins ce qui prescrit absolument ceci ou cela que ce qui interdit de croire trop absolument aux prescriptions de la coutume ou de la loi, comme de suivre trop aveuglément les impulsions de notre corps ou de la nature. Elle n'abolit pour autant ni les unes ni les autres, et ne saurait à elle seule tenir lieu de morale ${ }^{35}$ ". C'est parce qu'elle peut être symbolisée par une girouette se mouvant au gré des vents, que la raison, propre à justifier tout et son contraire, ne peut rien fonder d'universel si ce n'est l'universalité d'une raison essentiellement malléable. Comme l'écrit Montaigne, "cette belle raison humaine [...] brouille et confond le visage des choses selon sa vanité et inconstance» (II, XII, 580; PI. 564-565). La raison est dans tous les camps, même dans celui des fascistes. C'est pourquoi Marcel Conche a tort de croire, selon son élève, qu'elle seule peut les confondre. L'histoire nous a prouvé l'inverse. Universelle en droit, elle ne peut l'être en fait : «il n'y a pas (en tout cas pour l'homme) de raison pure, et tout jugement est historique ${ }^{36}$ ". Et si nous n'avons pas de fondement objectif pour justifier nos actions, sommes-nous pour autant bornés à l'inaction comme le sage d'Ariston? La réponse d'André ComteSponville est ici capitale et vaut, malgré sa longueur, d'être

34. "Montaigne cynique? (Valeur et vérité dans les Essais)", op. cit., p. 102.

35. Ibid.

36. Ibid., p. 103. Marcel Conche pourrait rétorquer à son disciple que sa différenciation entre le rationnel et le raisonnable le met à l'abri d'une telle critique. Cf. notre "Pour une nouvelle philosophie française : Marcel Conche», op. cit. 
citée en entier : "A-t-on besoin d'un fondement pour avoir, comme Montaigne, «horreur de la cruauté» (III, VIII, 922; PI. 900)? A-t-on besoin d'un fondement pour haïr, comme Montaigne, "toute sorte de tyrannie, et la parlière, et l'effectuelle» (III, VIII, 931; PI. 910)? A-t-on besoin d'un fondement pour se sentir, comme Socrate ou Montaigne (I, XXVI, 157; PI. 156; III, IX, 973; PI. 950), citoyen du monde et solidaire de tous les hommes? A-t-on besoin d'un fondement pour aimer la vie, la vérité et la paix? Et cette horreur, cette haine, ce sentiment, cet amour ne suffisent-ils pas - éclairés par la raison, guidés par la raison, mais non fondés sur elle - pour condamner et combattre le nazisme? Marcel Conche m'objecte alors que s'il n'y a pas de morale universelle, un nazi peut arguer de ses propres valeurs pour justifier ses actes. Sans doute, mais n'est-ce pas aussi ce qui se passe? En quoi cela me donne-t-il tort, d'un point de vue théorique? En quoi cela m'empêche-t-il, dans la pratique, d'opposer mes valeurs aux siennes, et ma force à sa force? Le relativisme de Montaigne, tel que je le lis, n'en fait pas plus un nihiliste, dans l'ordre pratique, que son scepticisme, dans l'ordre théorique, n'en fait un sophiste : l'incertitude n'empêche pas d'aimer la vérité; la tolérance n'empêche pas de haïr l'intolérance, ni de la combattre ${ }^{37}$ ".

Cette longue citation met à mal la tentative de fondation de la morale voulue par Marcel Conche. C'est bien là que l'élève se sépare du maître avec respect et amitié. Bien que la radicalité de leurs positions les éloigne l'un de l'autre, Montaigne reste le pont qui les relie et les réunit. C'est à travers lui qu'ils s'opposent et c'est en lui qu'ils se retrouvent. Qu'un tel homme ait vécu, en vérité le plaisir de philosopher sur cette terre en est augmenté!

\section{Sébastien Charles \\ Université d'Ottawa}

37. Ibid., p. 104. 


\section{À propos de Montaigne : bibliographie de Marcel Conche et d'André Comte-Sponville}

\section{Marcel Conche :}

- Montaigne ou la conscience heureuse, Paris : Seghers, 1964; $3^{e}$ éd., 1970; $4^{\circledR}$ éd., Éd. de Mégare, 1992.

- Montaigne et la philosophie, Treffort, Éditions de Mégare, 1987; $2^{e}$ éd., 1992; réimpression augmentée aux P.U.F., coll. «Perspectives critiques», 1996.

- «L'homme sans définition : Introduction à la philosophie de Montaigne", Revue de l'enseignement philosophique, octobrenovembre 1969, p. 1-24; repris sous le titre «L'homme sans définition" dans Montaigne et la philosophie.

- "La méthode pyrrhonienne de Montaigne», Bulletin de la Société des Amis de Montaigne, avril-décembre 1974, p. 47-62; repris sous le titre "Le pyrrhonisme dans la méthode" dans Montaigne et la philosophie.

- “Montaigne et l'ardeur de la vie», Bulletin de la Société des Amis de Montaigne, janvier-mars 1975, p. 5-21; repris sous le titre "Le pari tragique" dans Montaigne et la philosophie.

- "Le temps dans les Essais", Bulletin de la Société des Amis de Montaigne, janvier-juin 1978, p. 11-28; repris sous le titre "Le temps, la mort, l'ignorance" dans Montaigne et la philosophie.

- "Montaigne et le plaisir», Bulletin de la Société des Amis de Montaigne, janvier-juin 1979, p. 5-35; repris sous le titre «Plaisir et communication" dans Montaigne et la philosophie.

- "La découverte de la conscience morale chez Montaigne», Bulletin de la Société des Amis de Montaigne, janvier-juin 1981, p. 11-28; repris sous le titre "La conscience» dans Montaigne et la philosophie.

- "L'unité du chapitre Des coches», Études montaignistes en hommage à Pierre Michel, publiées par C. Blum et F. Moureau, Paris : Honoré Champion, 1984, p. 89-94; repris dans Montaigne et la philosophie.

- «Montaigne», Dictionnaire des philosophes, publié sous la direction de D. Huisman, P.U.F., 1984, p. 1843-1851.

- "La personnalité philosophique de Montaigne», Revue d'histoire littéraire de la France, no 5, 1988, p. 1006-1113.

- "Montaigne me manque», Montaigne philosophe, textes réunis par A. Comte-Sponville, Revue internationale de philosophie, $\mathrm{n}^{\circ}$ 181, 2, 1992, p. 157-168; repris dans Montaigne et la philosophie.

- "La signification de Dieu pour Montaigne", Bulletin de la Société des Amis de Montaigne, juillet-décembre 1993, p. 21-34; repris sous le titre "La signification de Dieu» dans Montaigne et la philosophie.

- Préface à P. Leschemelle, Montaigne ou le badin de la farce, Paris : Imago, 1995. 


\section{André Comte-Sponville :}

- "Je ne suis pas philosophe», Montaigne et la philosophie, Paris : Honoré Champion, coll. "Études montaignistes», 1993.

- "Montaigne ou la philosophie vivante", Literaturum II, Helf 4, Marburg, Dr. Wolfram Hitzeroth Verlag, 1989; repris dans Une éducation philosophique, Paris : P.U.F., coll. «Perspectives critiques», 1989.

- Préface au chapitre IX du livre III des Essais : De la vanité, Paris : Rivages, 1989.

- "Morale et politique dans les Essais", Montaigne, maire de Bordeaux, Mairie de Bordeaux, Éd. L'Horizon chimérique, 1992, p. 101-102.

- «N'oubliez pas Montaigne, svp!», L'Événement du jeudi, semaine du 6 au 12 février 1992.

- "Montaigne cynique?", Revue internationale de philosophie, no 181, 2, 1992, p. 234-279; repris sous le titre «Montaigne cynique? (Valeur et vérité dans les Essais)" dans Valeur et vérité, Études cyniques, Paris : P.U.F., coll. «Perspectives critiques», 1994. 